\title{
FROM HUMAN BODY DIGITIZATION TO INTERNET OF BODIES TOWARD A NEW DIMENSION OF MILITARY OPERATIONS
}

\author{
Vasile Florin POPESCU \\ Ministry of National Defense, Bucharest, Romania \\ popescuveve@gmail.com
}

\begin{abstract}
Digitization of the human body, philosophically said, - the "mating" with technology, represents the fusion of electronic technology with the human biology, which reduces the barriers of physical, digital and biological life. "The Internet of bodies", that is the imminent development of the field of digitization of the human body on a large scale, is the inevitable future of technology at this moment. Instead of devices connected to the Internet as in Internet of Things (IoT), human bodies can be connected to a network, with the potential to be controlled and monitored remotely. The Internet of bodies is from the author's point of view the future of technology, but this future is not so far away.
\end{abstract}

KEYWORDS: body digitization, digital and biological world, internet of bodies, subcutaneous devices

\section{Introduction}

The world of electronics is developing very rapidly and today there are many avant-garde ideas and projects, already under study that will be put into practice in a short time horizon. The purpose of this paper is to draw attention to a new dimension of military operations in the coming decades using advanced technologies integrated into the human body. This study represents a totally new futuristic approach that seeks to transcend conventional boundaries. The study is the result of a desk study that analyzed various sources of information in the field of new embedded technologies. This research is intended to sensitize military leaders and scientists to the development of a new technology integrated into the human body and how can be exploited. The author had this objective because he considers the concept of "digitization of the human body" would most likely involve a crucial transformation of military operations.
The research method is a qualitative one and is based on theories, personal approaches, case-situation description, interpretation, formulation of some findings; the possibility of generalization is questioned, given the small number of cases, small-scale research; however, there are possibilities for generalization.

\section{Subcutaneous Devices - Fusion Between Electronics and Biology \\ The electronics revolution has begun} in 1947 with the manufacture of the silicon transistors and semiconductors technology. Nowadays, we are assaulted by different and complex electronic devices necessary for everyday life. For almost 5 decades, silicone technology was the only option for electronics devices, but the current evolution of science has recalled the new trends that will be the subject of research and development in the coming decades. The discovery of graphene opened a new path to two-dimensional electronics device 
because of the high thermal and electrical conductivity and the mechanical flexibility of the two-dimensional hexagonal monoatomic lattice. Graphene represents the most durable material that has been ever tested. This may have marked the beginning of a 2D revolution in electronics - the allotropic thick of silicon, phosphorus and tin have the same structure as the cellular graphics, but their different properties make them suitable for other applications. Of course, all four have the ability to modify electronic components as we know them, which allows us to miniaturize them, increase efficiency and reduce costs. Many companies as Samsung, Huawei and Apple are already developing graphene applications. The development of conductive polymers was another important step. The 2000 Nobel Prizes, awarded by Alan J. Heeger, Alan MacDiarmide and Hideki Shirakawa, showed that plastics can lead to electricity (Royal Swedish Science Center, 2000). All these conducted to the production of organic electronic materials from carbon particles or polymers through chemical synthesis. They still do not compete with their traditional counterparts, but give advantages such as low cost, mechanical flexibility and biocompatibility, making organic electronics the optimal choice for a large number of applications.

High-tech commercially available products that rely on organic semiconductors, such as curved screen TVs, smart phone screens and portable solar cells, have already demonstrated their industrial maturity. Surely, the organic semiconductor market will grow exponentially over the next period of time.

In the 70's, apart from the capacitor, resistor and inductor, the electronics engineers came up with a new circuit element, called the memristor (memory + resistance). Hewlett Packard's engineers saw in 2007 its fantastic potential, and since then, many other scientists considered that electronics world entered in a new technology era, called the "ionic era".

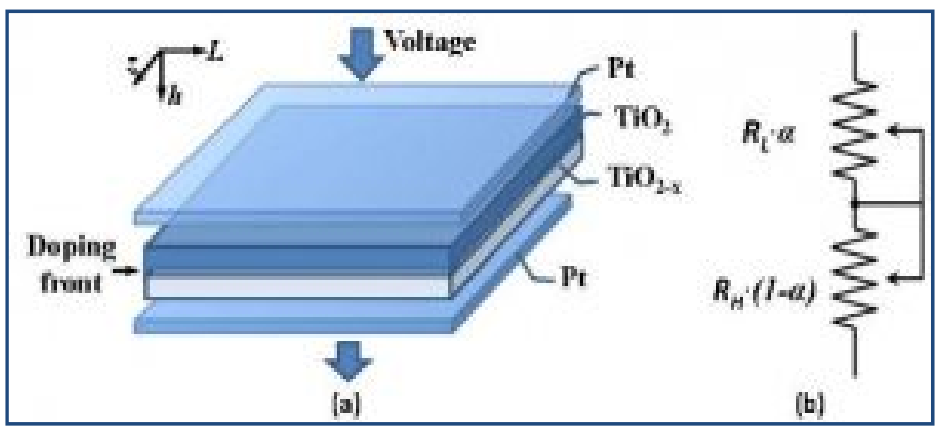

Figure no. 1: Memristor

(Source: Schematic model of the HP memristor, 2019)

Traditional transistors work with electron flux, while memristors combine electrons with ions or charged atoms. At the transistor level, all information is lost when the electron flow is interrupted (for example, by shutting down the system). Instead, the memristor "remembers" and stores information about the task it performs even if it is not already running. As a result, they promise to pave the path for better and safer storage of information. At the same time, memristors could provide safer and more efficient storage devices. In this way, there will not be lost information and the computers can be turn on and off immediately.

"Spintronic" - the use of the basic property of particles known as "electron spin" to process information is part of the same innovation field. By transferring information through charge and electron rotation, the device can acquire more diverse functions. 


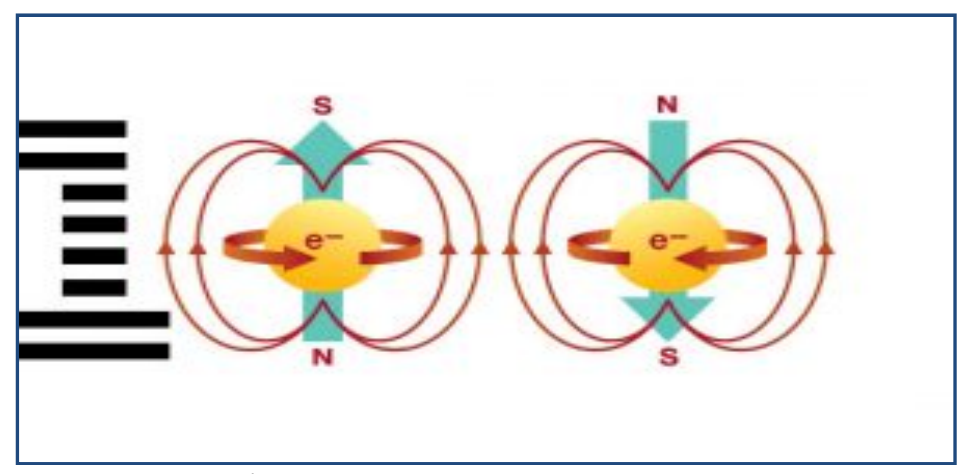

Figure no. 2: Spintronic

(Source: Spintronic, 2019)

Spintronic technology is currently being tested on storage devices such as hard drives, but is generally promising for digital electronics: higher computing power, higher data rates and increased storage capacity of information. Spintronic devices can be broken down, more flexible and stronger than their "parents" based on silicon.

Miniaturization of the electronics elements represents the crucial goal of electronic circuits. The nanotechnology industry, known as "mono-molecular electronics", uses single molecules or groups of single molecules as electronic "raw materials". This type of approach involves replacing bulky equipment, even existing hard equipment, with individual molecules. The smaller the amount of electronic components, the less the power consumption and sensitivity (and even performance) of the device.

It may also result in mono-molecular electronics of self-assembly, a phenomenon in which system components connect spontaneously to each other due to interactions or environmental factors to create a larger functional unit. Different solutions for molecular electronics are tested, including single-molecule wires and transistors. All of these attempts to find solutions are still in the lab phase, but they will soon pass this phase and will lead to a radical change in the world we live in.

Today, progress and invention are at an incredible speed. This is because we are no longer happy with today's progress, but we still want to be more attractive. But everyone is worried. What happens next? Fitbit has designed New Design, and is currently working on a new project called Skin. A smart digital tattoo that could be implanted under the skin of our hand which interacts with people's electronic devices. When working with the body's electrochemical energy, the device is always running and only works in the hands of the owner.

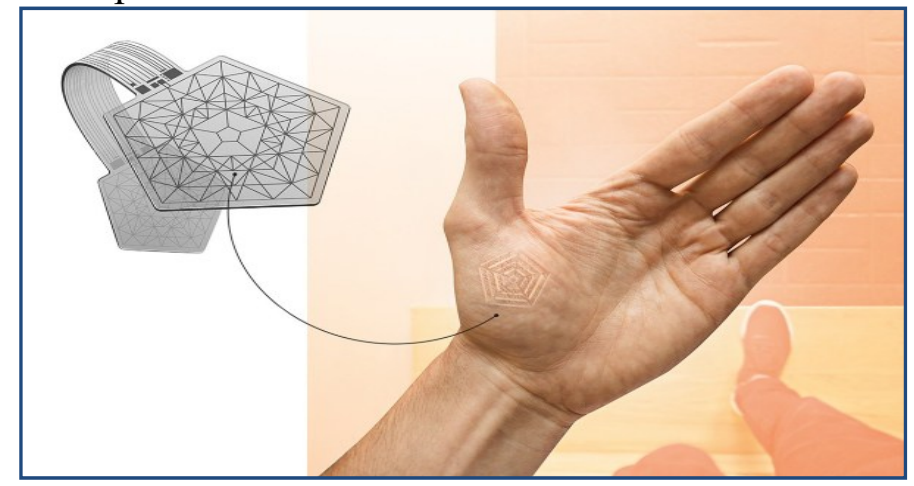

Figure no. 3: Subcutaneous devices

(Source: Adam, 2014) 
Moreover, these devices can provide information to the carrier in two ways: it can present the image in a discreet manner, or can take into account the gestures made by the owner, accepting the communication between himself and the other person. "When I started working on this project, everyone was a little grossed out by the idea of implanting something. There are lots of cultural boundaries" explains Jaeha Yoo, director of design company New Deal Design. "Of course, there are tattoos, piercings, people implanted birth control devices. These things are happening now. There is not a huge step to implant a subcutaneous" (Adam, 2014).

New Deal Design Company is confident that it could build the device subcutaneously in the next five years, given the current state of electronic research. The most difficult work will require flexible screen rather than the sensor, communication, implant or using body energy. Gadi Amit says even that we will soon see devices bearing subcutaneous: "Honestly, I can tell you that last year we discussed with business people, probably 3-4 times about physical invasive devices bearer ... it is a reflection on how the design will be over almost 10 years - not necessarily in the subject - but the weave biology, medicine, electronics, lots of interaction and cultural wisdom" (Fitbit, 2016).

\subsection{Computers that Dissolve in the Human Body}

Another avant-garde idea for scientists was represented by the dissolution of ultra-thin electronic devices in the human body, after they have completed their mission. This type of electronic solvents consists of silicon oxide and magnesium oxide in a protective silk layer. The silicone dissolves in water, but in a very long time. As a result, the researchers used very thin silicone sheets, called nano-membranes, to dissolve within a few days or weeks.

\subsection{Smart Tattoo Authentication}

The use of smart tattoos to authenticate tablets and phones remains another challenge to the technological evolution of the times we live in. In this regard, Regina E. Dugan, Special Project Manager for Motorola Mobility and former head of the Department of Defense's Advanced Research Project, said: "We believe there are many authentication options or trinkets, but I strongly take in consideration the authentication method that you can wear on your skin. We are talking about an electronic tattoo. Teens may not want to wear a watch, but you can be sure they wear a tattoo just to defy parents" (Dugan, 2013a).

Daily Mail Online (2014) states in article that: "MC10 tattoo, known as biostamps, helps medical staff to evaluate patients or works remotely, without the need for expensive large equipment. Motorola states that circuits with embedded antennae and sensors can be adapted to operate with mobile phones and tablets. Mobile devices could then be used to verify the identity of the owner and to enter the account automatically. This may prevent thieves and others from accessing the phone application, etc...if it is lost or stolen". Another Motorola authentication idea is Motorola Pill, also called vitamin identification, which is a pill that emits a signal authentication on 18 bits that can connect the person to devices. "Vitamin" containing a chip, it is swallowed and once arrived in the stomach, gastric acid serves the electrolyte position and activates the chip. Regina Dugan presents this technology as a real magic: "That means it will be my first superpower. I really want this superpower, which means my hands are like threads, and when I reach the phone, computer, door, car, they are directly recognized" (Dugan, 2013b). 


\section{Internet of Bodies}

The term Internet of Things (called IoT) entered the lexicon in 1999. Since then, there has been a proliferation of related terms, such as Web of Things (WoT) and Internet of Medical Things (IoMT) and so on. Lately, with the publication of the paper "Human digital immortality. Where Human Old Dreams"(Popescu \& Scarlat, 2017), a new concept associated with the concept of human body digitalization has attracted my attention. This is the concept of Internet of Bodies (IoB).

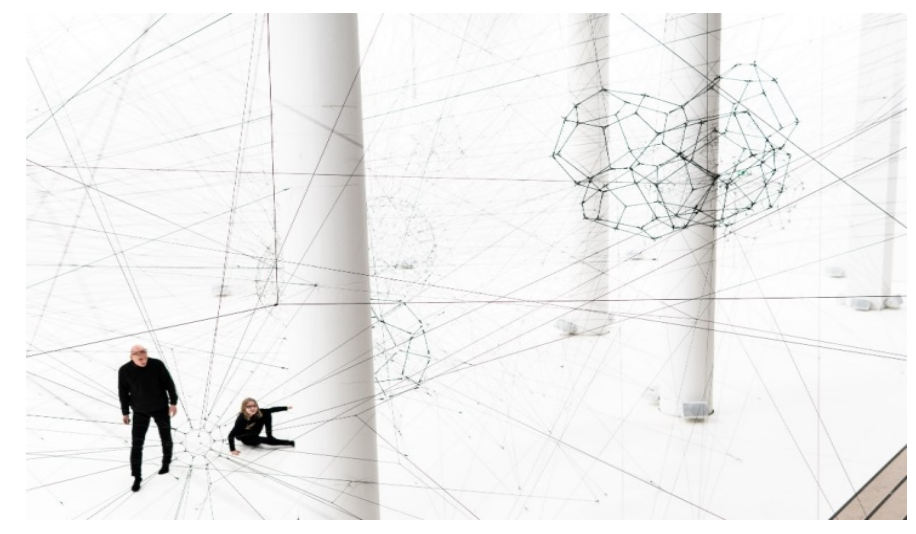

Figure no. 4: IoB - connected people

(Source: Sailesh, 2019)

The "Internet of bodies", that is the imminent development of the field of digitalization of the human body on a large scale, is the inevitable future of technology at this moment. Instead of devices connected to the Internet as in IoT, human bodies can be connected to a network, with the potential to be controlled and monitored remotely. The Internet of bodies is from the author's point of view the future of technology, but this future is not so far away.

The "Internet of bodies" begins to transform into a concept that describes the next generation of Internet of Things - the transition from a collection of everyday objects connected to the Internet to those devices attached to or embedded in the human body in order to collect and provide a constant flow of information. These devices could be part of a process of medical treatment, research or why not in the military domain.

The IoB could be broadly classified into two generations:

- First Generation - is the external type of the human body that includes devices such as Apple watches and Fitbit smart bands and others.

- The next generation is the internal type of the body, which has the aforementioned advances, such as the implants that come under it, the sensors embedded inside your skin.

Smart skin circuits that are capable of transforming the peripheral nervous system into an interface, cyber-contact lenses, millirobots, digital tattoos, or pay-per-view implants are some of the ways that are being developed to improve the human body. These innovations, which merge the human body and technology, make the barrier between man and machine somewhat more diffuse, leaving some ethical and moral questions. How can you ensure ethical use of this data? Who controls the use of personal data of our body? How is it possible to generate a situation where we can all benefit? In any case, it is not a trivial problem, because these changes could affect us as a society and could affect even the following generations, those of our children and grandchildren. 
The basic pillar of this theory is that the body becomes the center of digital interaction.

Thus, the physical space is mixed with the virtual one and both worlds are enriched. These immersive domes, which integrate different multimedia and interactive systems, are gradually expanding and it is expected that in the coming years it will become a reality.

The "Internet of bodies" will be something different from what we have now. The Internet of bodies is a new paradigm in which there will be values and benefits such as pleasure, intimacy, joy, compassion, hope, empathy, etc.

In full fever of selfies and likes, there will certainly be total connectivity of the human race.

\section{Toward a New Dimension of Military Operations}

Historically speaking, military research has held the monopoly of innovation and generated much of the cutting-edge technologies. But gradually, innovative products, such as the Internet or GPS, have been taken over and adapted for civilian use.

The modern capabilities of detecting the enemy and high precision weapons force the military to have high mobility and quick decision making. To do this, we need to have information from different sources in real time to be able to be disseminated promptly with all units involved in the operation, and one of the ways to solve this problem is to use Internet of Bodies (IoB) solutions. .

The use of the concept of Internet of Bodies (IoB) in the armies of many countries of the world will become a technological trend, a kind of indicator of the modernity and innovation of their armed forces.

However, most of the military infrastructure continues to be represented by "traditional" equipment such as tanks, ship destroyers, cruise missiles and intercontinental ballistic missiles, an impressive arsenal of nuclear bombs globally, nuclear-powered submarines or fighter jets. It can be appreciated that a maximum threshold has been reached in terms of the destructive force that the current military technique can generate, and research and production in the military industry are oriented towards the development of other offensive means that would neutralize the defense capabilities of an enemy state. The integration of new technologies into the military component leads, implicitly, to changing the essence and nature of conflicts. There has been talk of conventional, nuclear, asymmetrical wars, and more recently hybrid and informational warfare. Why not think about "intelligent wars" in which the concepts of digitization of the human body and, implicitly, the internet of human bodies are implemented?

This new scene of the $\mathrm{IoB}$ is an interesting one for the military sphere. An arms race in the technological world could begin, the army with the most advanced IOB technology being the "spearhead" of mankind. As the armies become more and more advanced due to technological advances, so too does the potential of an enemy to inflict damage by infiltrating the system and damaging it from within. It is possible to see wars fought on a digital level, as both sides will try to get into each other's IoB defense to deactivate their equipment. 


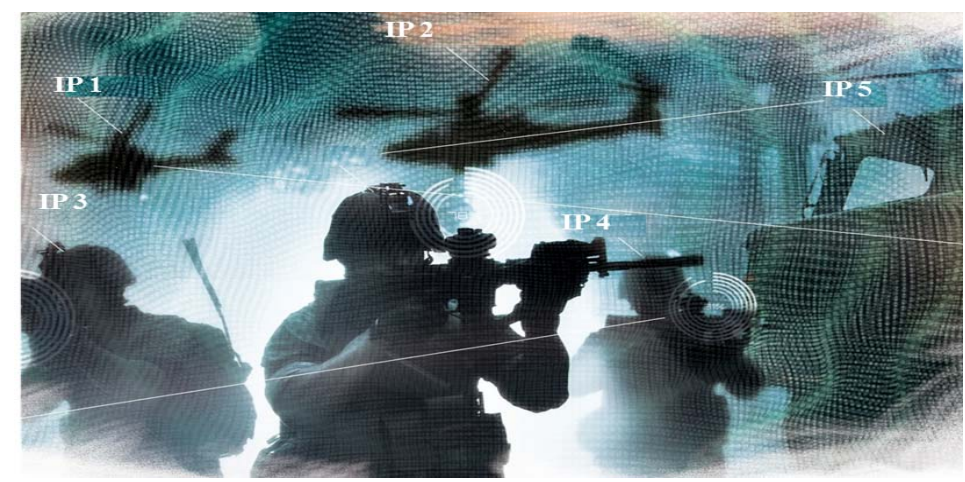

Figure no. 4: IoB on the battlefield

(Source: Author)

Future military operations will be based on interconnected soldiers, in order to gain superior defense capabilities. The IoB will connect soldiers with intelligent technology in weapons, radios, weapons and other objects, to provide troops with the "extra sensory" perception, provide situational understanding, equip combatants with predictive powers, provide better risk assessment and will develop shared intuitions.

Bringing the $\mathrm{IoB}$ to the military reality requires a broad partnership effort between authorities, industry and the scientific community. Progress in the technical disciplines, IT, software engineering must continue. But there are some philosophical questions around the resistance, continuity of operations and disaggregation of the capabilities to implement a military IOB.

\section{Conclusions}

The ability of 21 st century armies to understand, predict, adapt and exploit Internet of Bodies (IoB) on the future battlefield is essential to maintaining and increasing their competitive advantage. Most probably, future military operations will rely on interconnected soldiers to gain superior defense capabilities. The IoB will connect soldiers with smart technologies to give troops an "extra sensory" perception, provide superior situational understanding, equipped combatants with predictive powers, provide better risk assessment and develop common insights. However, as opposed to "civilians", IoB is subject to more serious risks due to participation in confrontations between different parties in the war.

\section{REFERENCES}

Adam, C. (2014). Is this interactive tattoo the future of 'wearable' technology? Fitbit designers plan interactive inking implanted in the skin. Daily Mail Online, available at: www.dailymail.co.uk/news/article-2798263/is-interactive-tattoo-future-wearable, accessed on 03 July 2019.

Dugan, R. (2013a). Wall Street Journal, available at: https://www.wsj.com/ video/regina-dugan-at-d11-badass/60D5FF10-B73E-48A7-92B4-49D23A18A78A.html, accessed on 21 July 2019.

Dugan, R. (2013b). Apple, Motorola Look to New Authentication Methods, available at: https://www.patentlyapple.com/patently-apple/2013/06/motorola-to-challenge-apples-futurefingerprint-authentication-with-electrical-vitamins-and-tattoo-superpowers.html, accessed on 21 July 2019. 
Fitbit (2016). Fitbit Tops the List as the Most Worn Wearable Globally, available at: www.digitalhome.ca/2016/05/fitbit-tops-the-list-as-the-most-worn-wearable-globally, accessed on 17 July 2019.

Popescu, F., \& Scarlat, Cz. (2017). Human Digital Immortality: Where Human Old Dreams and New Technologies Meet. IGI Global, Pennsylvania (USA), available at: www.igi-global.com/book/research-paradigms-contemporary-perspectives-human/166732, accessed on 04 August 2019.

Sailesh, A. (2019). Internet of Bodies - An Overview. Medium Corporation, IEEE LINK, available at: https://medium.com/ieeekerala/internet-of-bodies-an-overview9302579af62c, accessed on 03 July 2019.

Schematic model of the HP memristor. (2019), available at: https://www.researchgate. net/figure/Schematic-model-of-the-HP-memristor fig1_265474785, accessed on 03 July 2019.

Spintronic. (2019), available at: https://www.ibm.com/ibm/history/ibm100/us/en/, accessed on 06 July 2019.

The Royal Swedish Academy of Sciences. (2000). The Nobel Prize in Chemistry, 2000: Conductive polymers, available at: https://www.nobelprize.org/prizes/chemistry/2000/pressrelease/, accessed on 06 July 2019. 